\title{
CONOCIMIENTO DE BASE PARA EL DESARROLLO DEL TURISMO CIENTÍFICO EN LA PATAGONIA CHILENA ${ }^{1}$
}

\author{
Adriano Rovira Pinto \\ Universidad Austral de Chile \\ https://orcid.org/0000-0002-9951-8250 \\ Daniela Quintana Becerra \\ Universidad Austral de Chile \\ https://orcid.org/0000-0001-8605-6520
}

\section{RESUMEN}

El turismo de intereses especiales ha tenido un fuerte incremento en la Patagonia chilena, siendo incorporado como uno de los pilares del desarrollo regional. Dentro de este tipo de turismo, aparece con particular énfasis el turismo científico. En este trabajo se ha revisado el repositorio de tesis del sistema de bibliotecas de la Universidad Austral de Chile para analizar su contenido relacionado con la Región de Aysén, Patagonia chilena. Específicamente se pone atención en los aportes de las tesis de título y grado, al conocimiento que sirva de aporte al turismo científico. La información ha sido geo-referenciada con el fin de detectar la concentración de estudios, así como la carencia de antecedentes científicos que puede afectar a sectores específicos de la Región. De esta forma se pueden identificar los territorios sobre los cuales existe suficiente información, y aquellos en que es necesario iniciar investigaciones específicas.

Palabras Clave: Turismo científico; Aysén; Universidad Austral de Chile; tesis, bibliografía georeferenciada.

Fecha de recepción: 9 de mayo de 2018.

Fecha de aceptación: 11 de enero de 2019.

Instituto de Ciencias de la Tierra. Universidad Austral de Chile. Av. Eduardo Morales Miranda, s/n. Edificio Emilio Pugín, Of. 316. Campus Isla Teja. VALDIVIA (Chile).E-mail: arovira@uach.cl, daniela.quintana.b@ gmail.com

1 Proyecto ECOS-CONICYT C15H01. Geo-bibliografía de investigaciones científicas como herramienta para la mediación turística. Investigador principal Francisco Mena (CIEP) 


\title{
Basic knowledge for the development of scientific tourism in the Chilean Patagonia
}

\begin{abstract}
The especial interest tourism has had a strong increase in the Chilean Patagonia, being incorporated as one of the main objectives of the regional development. Inside this type of tourism, the scientific tourism appears with particular emphasis. In this work there has been checked the repository of thesis of the system of libraries of Universidad Austral de Chile to analyze his content related to Aysén's Region, specifically putting on attention in the contribution of the theses to knowledge supporting the scientific tourism. The information has been geo - referenced in order to detect the concentration of studies as well as the lack of scientific knowledge that can concern specific sectors of the Region. From this there can be identified the territories on which sufficient information exists, and those in which it is necessary to initiate specific investigations.
\end{abstract}

Key words: Scientific tourism; Aysén, Universidad Austral de Chile; thesis; geo-referenced bibliography.

\section{INTRODUCCIÓN}

El hecho de que el turismo es una de las actividades de más rápido crecimiento en el mundo, es algo definitivamente establecido según datos de la Organización Mundial del Turismo. La tendencia creciente de expansión de esta actividad se ha mantenido desde los años sesenta del siglo XX. Es así como en el 2017 las llegadas de turistas internacionales llegaron a 1.322 millones de personas, un $7 \%$ más que en el año anterior. Por su parte los ingresos generados por el turismo siguieron la misma tendencia a nivel mundial. En el caso del continente americano, la llegada de turistas internacionales creció un 3\% el año 2017, alcanzando los 207 millones. Este crecimiento fue aún mayor en América del Sur, en cuyo caso el incremento respecto al año 2016 fue del 7\% (Barómetro OMT del Turismo Mundial'2).

Desde el punto de vista económico, este crecimiento se traduce en un $9 \%$ del Producto Interno Bruto (PIB) del mundo, siendo responsable de alrededor del $10 \%$ del empleo mundial. En el caso de América, donde se concentra el 22\% de los viajes del mundo, el turismo representa el 8,6\% del PIB, contribuyendo a un 9,3\% del empleo regional (Chile, Subsecretaría de Turismo, 2015).

\subsection{Turismo en Chile}

Chile es un participante menor en el concierto mundial del turismo, aportando sólo con un $0,3 \%$ de las llegadas internacionales. Sin embargo para el país se constituye en un

2 http://media.unwto.org/es/press-release/2018-01-15/resultados-del-turismo-internacional-en-2017-losmas-altos-en-siete-anos 
sector económico relevante, generando el 3,2\% del PIB y del empleo nacional. Al considerar los efectos indirectos, esta participación asciende al 8,6\% (Chile, Subsecretaría de Turismo, 2015).

Según la estrategia Nacional de Turismo 2012 - 2020, "Durante el 2011, sobre 3 millones de turistas internacionales llegaron a Chile, un $11 \%$ más que en el año 2010" (Gobierno de Chile, s.f, p.22). Para el año 2016, según información de la Subsecretaria de Turismo, consignada en el periódico "La Tercera"3, el ingreso de turistas extranjeros a Chile aumentó en un $28 \%$ respecto al año 2015, y en un $26 \%$ en el 2016, lo que marca una tendencia clara al crecimiento de la actividad ${ }^{4}$.

Otro aspecto a tomar en cuenta a la hora de caracterizar la situación actual del turismo tiene que ver con los cambios en las conductas de los turistas. Esto se manifiesta por ejemplo en lo que se refiere a las preferencias por determinados destinos, donde se aprecia una búsqueda por destinos considerados exóticos que permitan la aventura. Esto ha ido acompañado por una tendencia creciente de atracción de los destinos considerados como turismo de intereses especiales (Chile, Subsecretaría de Turismo, 2015). En este ámbito, el Plan Nacional de Desarrollo Turístico Sustentable plantea en su Línea de Acción 2, la necesidad de desarrollar nuevos destinos "mediante la ampliación de la oferta de servicios basados en líneas temáticas transversales que nos permitan ser más competitivos y destacar la oferta diferenciadora de nuestro país" (Chile, Subsecretaría de Turismo, 2015, p. 30).

Entre los tipos de turismo que el Plan plantea como posibles de desarrollar en Chile se encuentra el turismo de naturaleza, el turismo indígena, el turismo astronómico, el turismo científico, el enoturismo, turismo de negocios, turismo náutico y turismo deportivo. En este trabajo se analizará específicamente el caso del turismo científico.

\subsection{Turismo científico}

El concepto "turismo científico" comienza a aparecer en la literatura especializada, así como en los mensajes de operadores, a mediados de los años noventa del siglo veinte y busca principalmente involucrar a los turistas en el trabajo que desarrollan los investigadores en diferentes disciplinas, básicamente en contacto con la naturaleza y en lugares que además presentan algún grado importante de atractivo para ser visitados (Bourlon y Mao, 2011). De esta manera acerca al gran público a investigadores científicos o a lugares directamente vinculados con la ciencia, como museos, parques naturales o lugares de alto interés científico (García y Martínez, 2017).

Este tipo de turismo aparece ligado principalmente a países considerados como de mayor desarrollo, como una forma de incentivar la participación de las personas en el conocimiento de la naturaleza. García y Martínez (2017) lo consideran complementario al turismo de aventura, al ecoturismo y al turismo responsable. Logra poner en valor la naturaleza y acerca al turista a los lugares en una dimensión intelectual en la medida en que el turista es quien realiza la exploración científica.

3 La Tercera, 13/08/2016. http://www.latercera.com/noticia/visitas-de-turistas-extranjeros-a-chile-aumentan-un-28/

4 http://www.subturismo.gob.cl/wp-content/uploads/2015/10/20170731-TURISMO-RECEPTIVO2016-julio.pdf 
Bourlon y Mao (2011) plantean que el término turismo científico es usado para referirse a diversos tipos de actividades, ante lo cual los autores proponen la existencia de cuatro grandes tipos en que este turismo puede ser clasificado: i) el turismo aventura de dimensión científica; ii) el turismo cultural de contenido científico; iii) el eco-voluntariado científico; y iv) el turismo de investigación científica. García y Martínez (2017) por su parte, no consideran que el primer tipo mencionado forme parte del turismo científico. Existen también autores que incluyen dentro del turismo científico, el llamado turismo de congresos, específicamente congresos de índole científica (Sieber et al, 2015; Cynarski y Kubala, 2017).

El turismo aventura de dimensión científica y el de investigación científica, según Bourlon y Mao (2011) implican una importante participación del actor en el producto turístico, no así en el caso de los otros dos tipos. La posición que ocupa la temática científica en estas prácticas también es diferente, según el caso. Así, en el turismo de investigación científica y en el eco-voluntariado científico, el interés por los aspectos científicos ocupa una posición central, en tanto en los otros tipos es más bien de carácter periférico (Bourlon y Mao, 2011).

Pardo y Nieto (2015), en sus estudios respecto a la actividad turística que se desarrollan en la Antártida, concluyen que existe una relación de complemento entre el turista recreacional, turista de crucero en su definición, y el turista-científico, que permanece por meses en el territorio antártico desarrollando investigaciones.

National Geographic ofrece claros ejemplos de estos tipos de turismo en sus ofertas de subvenciones para "jóvenes exploradores", que permiten el desarrollo de proyectos de investigación, conservación y exploración ${ }^{5}$.

Los proyectos y actividades que se desarrollan en la Antártida constituyen un ejemplo de turismo científico en que se combinan los intereses de los científicos y de los viajeros de cruceros, generando un turismo sostenible, controlado, interrelacionado y permeable entre sí, con lo que se ha posibilitado el desarrollo y crecimiento de un turismo que favorece la investigación (en las bases antárticas) y la difusión del conocimiento (Pardo y Nieto, 2016). Los mismos autores señalan que en la campaña turística 2012 - 2013, se llegó a un total de 34.354 turistas comerciales, cifra menor al máximo alcanzado en 2007 - 2008, en que se llegó a los 46.069 turistas.

Como señalan Pardo y Nieto (2016), el turismo antártico presenta una doble perspectiva, por un lado el ocio en forma de ecoturismo centrado en la naturaleza antártica y por otro el turismo científico centrado en investigaciones que se desarrollan en las bases de los diferentes estados presentes. Ambos, el turismo vacacional y el científico se localizan en las áreas de mayor accesibilidad.

Sea cual sea el tipo de turismo científico, siempre se requiere de un soporte académico, de manera de ofrecer al turista la adecuada y suficiente información científica y técnica que haga de su experiencia algo satisfactorio y que cumpla plenamente con las expectativas.

Un tipo de turismo que ejemplifica esta necesaria disponibilidad de información académica, es el geoturismo, en el cual las ciencias de la tierra juegan un rol funda-

5 http://masoportunidades.org/convocatoria-national-geographic-jovenes-ambientalistas 
mental en dotar al visitante de los antecedentes indispensables para que su experiencia sea exitosa y satisfactoria. La promoción de este tipo de turismo basado en la visita a geositios y en la conservación de la geodiversidad permite al visitante acrecentar sus conocimientos sobre la importancia de las ciencias de la tierra (Comanescu y Dobre, 2009; Kuvaliková, 2013; Pralong, 2005; Reynard, 2008)

Los servicios de interpretación juegan un papel central en el éxito de la experiencia, como en el caso del Valle de Susa (Alpes italianos) en que los turistas pueden adquirir el conocimiento que les permita comprender la evolución geológica y geomorfológica de un territorio, más allá de la simple contemplación (Giordano, Giardino, Perotti, Ghiraldi y Palomba, 2016).

De la misma manera Migon y Pijet-Migon (2016) se refieren al desarrollo del geoturismo en geositios volcánicos en Polonia. En este caso los autores, a la par que analizan las potencialidades turísticas del área, proveen de abundante y sólida información científica. Esta información permitirá, según señalan, desarrollar buenos planes de turismo científico a la vez que implementar proyectos de geoeducación, destinados tanto a escolares como a público en general. Si bien el sector analizado tiene una tradición en el uso turístico de los mejores geositios, los autores señalan como necesario para fortalecer la actividad, traducir el conocimiento científico a una forma accesible para el público general, diseminar la información de manera de complementar el valor de estos sitios e implementar planes adecuados para la gestión de los sitios más relevantes de manera de evitar que sus valores escénicos y científicos se vean dañados por la actividad humana (Migon y Pijet-Migon, 2016).

Los conocimientos necesarios para comprender la complejidad que suelen presentar los geositios no siempre los poseen los turistas, las evidencias demuestran, según Reynard (2008), que los conocimientos en ciencias de la tierra deben ser cuidadosamente trabajados, para transformarlos en productos turísticos. Se debe poner especial énfasis en la producción de cartografía de alta calidad para las geo-guías y en el desarrollo de prácticas de difusión del conocimiento científico.

También en el caso del turismo antártico Pardo y Nieto (2015), destacan el hecho de que las investigaciones científicas que se realizan en la Antártida, servirán de base para el turismo de cruceros, permitiendo aportar con conocimientos para enriquecer la experiencia del turista, a la vez que apoyando los programas de protección ambiental y las buenas prácticas de un turismo responsable.

Siguiendo esta idea de la necesaria disponibilidad de información para sostener proyectos de turismo científico, este artículo analiza la producción científica de la Universidad Austral de Chile, a través de las tesis de título y grado realizadas en las diferentes escuelas de la Universidad. La Universidad Austral de Chile ha mantenido una constante presencia en la Región de Aysén. En 1993 formó el Centro de Investigación en el Manejo y Conservación de Recursos Naturales, Centro Trapananda, que ha apoyado el quehacer de los investigadores de la Universidad que por medio de diversos proyectos de investigación han contribuido al conocimiento de la Región de Aysén. El objetivo de este artículo es aportar los antecedentes disponibles en las tesis de los estudiantes de pre y post grado, de manera de ponerlos al servicio del desarrollo de proyectos de turismo científico. 


\subsection{Turismo científico en Aysén}

García y Martínez (2017) señalan a Chile como uno de los países que a nivel mundial presenta alta potencialidad para el desarrollo de turismo científico, atendiendo a la diversidad paisajística y a la existencia de importantes sitios de interés para la investigación. En este sentido, la Región de Aysén, en la Patagonia chilena, se alza como uno de los territorios destacados en este ámbito, concentrando una alta demanda por visitas a las áreas silvestres protegidas, tanto de turistas nacionales como extranjeros.

La Región de Aysén se localiza entre los $43^{\circ} 38^{\prime}$ y $49^{\circ} 16^{\prime}$ de latitud Sur y posee una superficie de $108.494 \mathrm{~km}^{2}$ (Figura 1). La población estimada al año 2016 es de 109.317 habitantes por lo que registra una densidad de alrededor de 1 habitante por $\mathrm{km}^{2}$, la más baja del país. La Región cuenta con un conjunto de atributos que la hacen privilegiada para el turismo de naturaleza y de aventura, dada la variedad de paisajes, un ambiente prístino y una alta biodiversidad, a lo que se suma una elevada belleza escénica, lo que la hace particularmente atractiva para los visitantes. Casi la mitad de su territorio está en el Sistema Nacional de Áreas Silvestres Protegidas del Estado (SNASPE), lo que le agrega mayor valor a su oferta de atractivos. Todo ello lleva a que el turismo se plantee como uno de los pilares básicos del desarrollo económico regional (Gobierno de Chile (b). s.f).

Esta oferta turística sustenta la cantidad de llegadas que registra la Región, alcanzando en la temporada de diciembre, enero y febrero 2015 - 2016, la cifra de 203.763 visitantes, un $20 \%$ más que la misma temporada del año anterior (SERNATUR, s.f. (a)). Silva (2002) proyectaba para el año 2020 un total de entre 422.000 y 861.000 turistas anuales para la región de Aysén.

Dadas las características territoriales de la Región de Aysén, los tipos de turismo que mayormente se identifican son el turismo cultural, el agroturismo, el turismo deportivo y el ecoturismo. Este último es el que adquiere mayor relevancia dado el creciente interés por el turismo de naturaleza. Esto queda además refrendado en los estudios de los perfiles de turistas que visitan la Región y cuyo principal interés lo constituyen sus Parques Nacionales (Silva, 2002).

En la práctica del ecoturismo resultan importantes las actividades de descubrimiento, observación, senderismo y de conservación de la naturaleza (Silva, 2002). Esto lleva a que este tipo de turismo sea el que más se relaciona con el turismo científico, según lo expresado en el acápite anterior.

En consonancia con todo lo anterior, SERNATUR establece como objetivo: "Promover el desarrollo turístico de la Región de Aysén, a través de la puesta en valor turístico de sus recursos naturales y culturales, el aumento de la demanda apuntando a los grupos objetivos de turismo de intereses especiales y el mejoramiento de la competitividad de las empresas del sector" (SERNATUR s.f. (b) p.23).

Respecto a iniciativas llevadas a cabo en este ámbito, destaca la labor desarrollada en el Departamento de Turismo Sustentable del Centro de Investigación en Ecosistemas de la Patagonia (CIEP) que a través de diversas iniciativas ha instalado el tema del turismo científico en la Región de Aysén. En este ámbito se puede mencionar el artículo de Bourlon y Mao (2011), en el cual proveen de una interesante revisión de actividades desarrolladas 
en la Región de Aysén y que pueden ser consideradas en alguna de las cuatro formas en que ellos reconocen al turismo científico.

Más recientemente, como resultado del proyecto Archipiélagos Patagónicos, Destino mundial para el turismo científico (Bourlon, Soto y Pastrian, 2016), se hace un detallado análisis del área litoral de Aysén y se aporta la información científica disponible que sustenta cada una de las propuestas. Dotado de una abundante información respaldada por la bibliografía especializada disponible, los autores recorren el litoral aisenino y presentan un conjunto de posibilidades de turismo, divididas en cinco zonas prioritarias, que de norte a sur son: Nómades y colonos de los canales australes; tres culturas y un mundo; una vida ligada al mar; un territorio de exploradores y el principio del fin del mundo.

\section{Figura 1 \\ ÁREA DE ESTUDIO. REGIÓN DE AYSÉN}

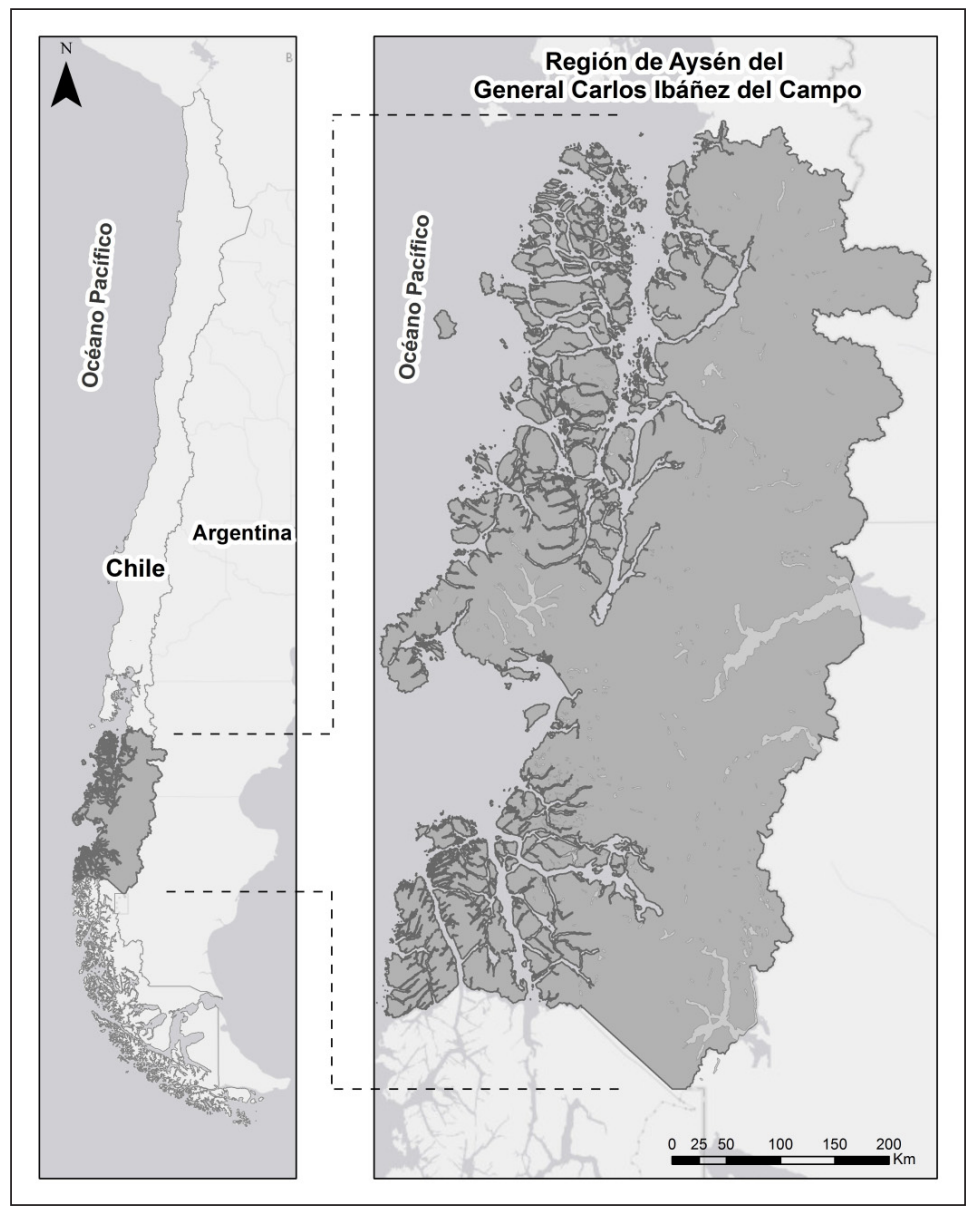

Fuente: Elaboración propia. 


\section{METODOLOGÍA}

La metodología aplicada en este artículo consistió en la revisión de material académico consistente en memorias y tesis de título y grado, elaboradas por estudiantes de pregrado y postgrado de la Universidad Austral de Chile que hayan realizado su investigación dentro del límite administrativo de la Región de Aysén, con el fin de clasificarlas según grupos temáticos y georreferenciarlas para su representación cartográfica. De esta manera se podrá evaluar el aporte que estas investigaciones pueden hacer al desarrollo del turismo científico en la Región de Aysén.

Para llevar a cabo este procedimiento se utilizó la propuesta metodológica de clasificación y sistematización generada por Territorio Aysén (2014), la cual consiste en crear una base de datos bibliográficos a partir de la recolección de material científico, rescatando la información más relevante de cada una de ellas (título, nombre de autor, resumen, bibliografía, coordenadas, entre otros) y asignándole al material recopilado un área científica y un grupo temático afín, obteniendo así una clasificación. De esta manera el informe de Territorio Aysén (2014), generó información relevante para los efectos del turismo científico, específicamente en el sector litoral de la región de Aysén.

Sin embargo, y para efectos de este trabajo fue necesario realizar modificaciones a la citada propuesta original, con el fin de adecuarla al contexto de estudio, por lo que el trabajo se dividió en tres etapas:

1. Consulta, obtención y clasificación del material bibliográfico

2. Creación de la base de datos bibliográficos

3. Creación de una Geodatabase y representación cartográfica.

En la primera etapa de consulta y obtención de los documentos, solo se consideró a las memorias de título y tesis. La consulta se generó mediante una búsqueda con palabras claves en el sistema virtual de bibliotecas de la Universidad Austral de Chile, además de consulta presencial, obteniéndose memorias y tesis tanto en formato impreso como digital.

Según la propuesta ya mencionada, la clasificación del material recolectado debe ser en primera instancia catalogado de acuerdo a la disciplina científica y luego clasificado en tres áreas temáticas afines (ver Tabla 1). De acuerdo a las investigaciones recogidas, se estableció una clasificación de las áreas temáticas según los siguientes criterios:

- Territorio y ciencias sociales: este grupo contiene a todas las disciplinas asociadas a la interacción del ser humano con el medio, ocupación del espacio y las relaciones intra-específicas que éste realiza a diario, considerándose áreas científicas como la planificación y ordenamiento territorial, ciencias de la ingeniería (construcción), ciencias económicas, y las ciencias de índole social como antropología, sociología, historia, ciencias de la comunicación y lingüística.

- Ciencias de la vida: dentro de este grupo se consideraron a las disciplinas asociadas a la naturaleza viva, hábitat y comportamiento de las especies como la biología marina, acuicultura, zoología, zootecnia, entomología, bacteriología, ecología, silvicultura y botánica. Así también se consideró dentro de esta clasificación a las ciencias de la salud, tanto para el bienestar humano como para el de las demás especies (medicina veterinaria). 
- Ciencias de la tierra: este grupo contiene a las disciplinas que se encargan de explicar el comportamiento y los distintos procesos de la naturaleza y la materia inerte. Entre ellas está la hidrología, climatología, geografía física, química y la agronomía (edafología).

\section{Tabla 1}

\section{SISTEMA DE CLASIFICACIÓN DEL MATERIAL BIBLIOGRÁFICO EN GRU- POS TEMÁTICOS Y ÁREAS CIENTÍFICAS}

\begin{tabular}{|l|l|}
\hline Grupo Temático & Área científica \\
\hline Ciencias Sociales y Políticas & $\begin{array}{l}\text { Antropología,Arqueología, Ciencias Políticas, Demografía, } \\
\text { Derecho, Etnología, Etnografía, Economía, Filosofía, } \\
\text { Historia, Literatura, Lengua, Lingüística, Psicología, } \\
\text { Sociología, Urbanismo y Ordenamiento Territorial. }\end{array}$ \\
\hline Ciencias de la Vida y Marinas & $\begin{array}{l}\text { Biología, Ecología, Ciencias del Suelo, Biomatemáticas, } \\
\text { Etología,Paleontología,Biología/Fauna Marina, Zoología, } \\
\text { Botánica (biología vegetal), Biofísica, Acuicultura, } \\
\text { Oceanografía, Biotecnología, Ciencias Atmosféricas } \\
\text { (Meteorología), Biología animal (Zoología), Bioquímica, } \\
\text { Biología celular, Biología de insectos (Entomología), } \\
\text { Biología Vegetal (Botánica), Biología /Fauna Terrestre, } \\
\text { Ornitología, Bioquímica, Pesquería, Biología Marina, } \\
\text { Zoología Marina. }\end{array}$ \\
\hline Ciencias de la Tierra & $\begin{array}{l}\text { Geografía, Geología, Geomorfología, Climatología, } \\
\text { Sismología, Paleontología, Geofísica, Edafología, } \\
\text { Hidrología, Meteorología. }\end{array}$ \\
\hline
\end{tabular}

Fuente: Modificado de Territorio Aysén (2014)

La Segunda etapa consistió en la sistematización y construcción de la base de datos bibliográficos para el almacenamiento de la información recopilada. La Tabla 2 presenta la lista de los campos que conformaron la base de datos.

La determinación del potencial turístico (Alto, Medio y Bajo) y el criterio de selección, se basó principalmente en el contenido de la tesis, en la localización y en el objeto de estudio. Un alto potencial se asignó a tesis, por ejemplo, que tuvieran un fuerte contenido turístico en su investigación, o que hayan sido realizadas en alguna reserva nacional o un sitio de interés para el turismo, o que el objeto de estudio haya sido una especie o una situación particular con alto atractivo turístico o con potencial para interesar a turistas en la realización o participación en proyectos científicos.

En cuanto a las coordenadas, los documentos recopilados representaban sus zonas de estudio con coordenadas geográficas por lo que fue necesario transformarlas a UTM. Para las tesis que solo indicaban la ubicación de forma verbal, fue necesario localizarlas a través de herramientas como Google Earth o mapas de la Región, y para las tesis que tenían zonas de estudio en distintos sectores de la Región, fue necesario determinar el área 
completa y dejar un punto central como ubicación del estudio. El mismo procedimiento se aplicó en el caso de tesis cuya área de estudio fuera la Región completa.

Tabla 2

CAMPOS DE LA BASE DE DATOS DE LA BIBLIOGRAFÍA ANALIZADA

\begin{tabular}{|c|c|}
\hline NID & Número Identificador. \\
\hline TÍTULO & Título del documento. \\
\hline AUTOR & Nombre del autor. \\
\hline PATROCINANTE & Nombre del profesor guía. \\
\hline ESCUELA & Carrera en la que se originó la memoria o tesis. \\
\hline ÁREA CIENTÍFICA & Disciplina científica a la que se asocia el documento \\
\hline GRUPO & Área temática afín. \\
\hline AÑO & Año de publicación del documento. \\
\hline REFERENCIA & $\begin{array}{l}\text { Materiales bibliográficos que sirvieron de referencia para elaborar } \\
\text { el documento. }\end{array}$ \\
\hline IDIOMA & Idioma en que está desarrollado el documento. \\
\hline POTENCIALTURÍSTICO & $\begin{array}{l}\text { Indica si el material tiene relación "Baja", "Media" o "Alta" con la } \\
\text { aplicabilidad de proyectos turísticos. }\end{array}$ \\
\hline CRITERIO SELECCIÓN & Indica los aspectos considerados para determinar el potencial. \\
\hline TOPÓNIMO & $\begin{array}{l}\text { Topónimo geográfico que hace referencia al contexto del } \\
\text { documento. }\end{array}$ \\
\hline URL & Dirección electrónica de localización del documento. \\
\hline RESUMEN & $\begin{array}{l}\text { Breve descripción de lo realizado en la memoria de título y tesis } \\
\text { (máximo } 200 \text { caracteres). }\end{array}$ \\
\hline MAPA & Localización de la zona de estudio de manera gráfica. \\
\hline ÁREA HA & Superficie en hectáreas del área de estudio del documento. \\
\hline LAT COOR_X & Coordenada "X" (Latitud) del centro del área de estudio (UTM). \\
\hline LONG COOR_Y & Coordenada "Y" (Longitud) del centro del área de estudio (UTM). \\
\hline HUSO & Huso horario \\
\hline
\end{tabular}

Fuente: Elaboración propia sobre la base de metodología propuesta por Territorio Aysén (2014)

La tercera etapa consistió en la elaboración de la base de datos geográficos o también conocida como GeoDataBase la cual es utilizada para representar los datos espacialmente en un software de Sistema de Información Geográfica. Para esto se utilizaron las coordenadas XY con las que se creó una red de puntos con la ubicación de las distintas memorias de título y tesis con los atributos determinados en la base de datos bibliográficos. Para 
estos efectos se utilizó el software QGis 2.18 y luego se exportaron los mapas a formato de publicación.jpg.

\section{RESULTADOS Y DISCUSIÓN}

Los resultados que se presentan a continuación corresponden al análisis de la base de datos alfa numérica y a la geodatabase, ambas elaboradas con el material recopilado en la base de datos del Sistema de Bibliotecas de la Universidad Austral de Chile. En total se analizan las características de 107 tesis o memorias de pre y post grado.

\subsection{Análisis de las tesis y memorias de título o grado}

Como se indicó anteriormente, este análisis comprende 107 memorias de título o tesis de pre y post grado, realizadas en alguna de las escuelas o programas de la Universidad Austral de Chile. La Tabla 3 contiene un detalle de las tesis, de acuerdo a los criterios de clasificación utilizados. De su información, se advierte que el Grupo Temático de mayor producción ha sido el que agrupa las disciplinas clasificadas como Territorio y Ciencias Sociales, con un total de 54 menciones. Ciencias de la Vida por su parte reúne 41 trabajos y Ciencias de la Tierra solamente 12 .

Respecto a las áreas científicas definidas se destaca Agronomía, en el caso de Ciencias de la Tierra, con 9 de las 12 tesis clasificadas en este grupo. Estas tesis presentan una clara orientación hacia temas relacionados con la ganadería y el manejo de praderas, temática relevante en la Región de estudio.

Tabla 3

DISTRIBUCIÓN DE LAS MEMORIAS Y TESIS SEGÚN GRUPO TEMÁTICO Y ÁREA CIENTÍFICA

\begin{tabular}{|l|l|c|}
\hline GRUPO TEMÁTICO & ÁREA CIENTÍFICA & NÚMERO DE TESIS \\
\hline CIENCIAS DE LA TIERRA & & $\mathbf{1 2}$ \\
\cline { 2 - 3 } & Agronomía & 9 \\
\cline { 2 - 3 } & Climatología & 1 \\
\cline { 2 - 3 } & Hidrología & 1 \\
\cline { 2 - 3 } & Química & $\mathbf{4 1}$ \\
\hline CIENCIAS DE LA VIDA & & 14 \\
\cline { 2 - 3 } & Biología Marina & 2 \\
\cline { 2 - 3 } & Botánica & 9 \\
\cline { 2 - 3 } & Ecología & 1 \\
\cline { 2 - 3 } & Etología & 4 \\
\cline { 2 - 3 } & Salud & 9 \\
\hline
\end{tabular}




\begin{tabular}{|l|l|c|}
\hline GRUPO TEMÁTICO & ÁREA CIENTÍFICA & NÚMERO DE TESIS \\
\hline \multirow{4}{*}{ CIENCIAS DE LA VIDA } & Silvicultura & 3 \\
\cline { 2 - 3 } SOCIALES & Zoología & 7 \\
\cline { 2 - 3 } & Zootecnia & $\mathbf{5 4}$ \\
\hline \multirow{5}{*}{\begin{tabular}{l} 
TERITORIO Y CIENCIAS \\
\cline { 2 - 3 }
\end{tabular}} & Antropología & 7 \\
\cline { 2 - 3 } & Arquitectura & 1 \\
\cline { 2 - 3 } & Ciencias de la Comunicación & 6 \\
\cline { 2 - 3 } & Economía & 11 \\
\cline { 2 - 3 } & Educación & 1 \\
\cline { 2 - 3 } & Historia & 4 \\
\cline { 2 - 3 } & Ingeniería & 1 \\
\cline { 2 - 3 } & Lingüística & 1 \\
\cline { 2 - 3 } & Oceanografía & 1 \\
\cline { 2 - 3 } & Planificación territorial & 6 \\
\cline { 2 - 3 } & Psicología & 1 \\
\cline { 2 - 3 } & Sociología & 6 \\
\cline { 2 - 3 } & Turismo & 1 \\
\hline
\end{tabular}

Fuente: Elaboración propia.

En el grupo de Ciencias de la Vida la mayor proporción la presenta Biología Marina con 14 trabajos, seguida de Ecología con 9 y Zootecnia con 7. Las tesis en Biología Marina presentan una concentración en temas relativos a especies de agua dulce y salmones, particularmente asociados al desarrollo de la pesca deportiva, temática que constituyó una de las áreas de trabajo en el Centro de la Trapananda de la Universidad Austral de Chile en Coyhaique. En ecología se puede apreciar un predominio de trabajos en ecología de bosques, en tanto en el caso de Zootecnia, predominan las tesis relacionadas con sanidad animal.

En el caso de Territorio y Ciencias Sociales, Economía registra 11 tesis, en tanto Planificación Territorial tiene 8 y Antropología 7 tesis. Con 6 tesis en este grupo se pueden mencionar también las áreas de Ciencias de la Comunicación, Ingeniería y Turismo. Las tesis de economía tienden a concentrarse en temas relacionados con la evaluación económica de proyectos o inversiones. En el caso de Planificación Territorial, la temática relativa a infraestructura registra la mayor proporción de trabajos, respondiendo a la relevancia que este tema ha tenido en Aysén. Las tesis de Antropología por su parte presentan una mayor diversidad pudiendo identificarse solamente una cierta tendencia a concentrarse en temas relativos a las relaciones entre comunidades locales y medio ambiente

Es necesario destacar la relativa baja participación de Ciencias de la Tierra y particularmente de las áreas científicas como geología, geomorfología, climatología e hidrología, 
pese a la relevancia que estas áreas de conocimiento tienen para la Región de Aysén. Este es un hecho que a la propia Universidad Austral de Chile le servirá para revisar su participación en la investigación científica de esta Región.

Sin lugar a dudas la presencia o ausencia de trabajos de tesis en las áreas científicas incluidas en este artículo, tienen relación con la actividad de las escuelas y carreras de la Universidad. Así por ejemplo, es de suponer que la reciente creación de las carreras de geografía y geología, repercutirá en una mayor cantidad de trabajos de titulación en disciplinas de Ciencias de la Tierra.

Para evaluar la participación que han tenido las carreras de la Universidad en la producción científica a través de las tesis, la Tabla 4 presenta la distribución de estos trabajos según carrera de pregrado, en tanto la Tabla 5 hace lo propio para los programas de magister.

En el caso de las carreras de pregrado, es interesante advertir la fuerte participación de las carreras de Biología marina y Medicina Veterinaria, con 18 y 10 trabajos respectivamente. A ellas les siguen Agronomía, Periodismo e Ingeniería Forestal con 9, 8 y 7 tesis respectivamente.

Respecto a los programas de post grado, fue posible encontrar tesis de grado en tres programas de magister. De ellos destaca el Magister en Economía y Gestión Regional con 6 tesis, el Magister en Desarrollo Rural aporta con 4 tesis y el Magister en Ciencias del Suelo con una. En este caso es importante señalar que tanto el Magister en Economía y Gestión Regional, como el de Desarrollo Rural, se dictaron extraordinariamente en Coyhaique, en una o dos versiones.

Para definir la realización de estos trabajos de titulación en función del tiempo, se ha preparado la Tabla 6 que da cuenta de la distribución de las tesis desarrolladas en periodos temporales a partir de 1970, año en que aparece la primera tesis registrada en el sistema de bibliotecas de la Universidad Austral de Chile, aplicada a una temática de la región de Aysén. En esta Tabla 6 se puede observar que, en el caso de Ciencias de la Tierra, la producción ha sido más o menos homogénea a través del tiempo. En cambio, en el caso de Ciencias de la Vida y de Territorio y Ciencias Sociales es posible apreciar una fuerte concentración en los años posteriores al 2000, particularmente en el periodo entre los años 2006 y 2010 , en ambos casos.

Tabla 4

NÚMERO DE TESIS SEGÚN CARRERA DE LA UNIVERSIDAD AUSTRAL DE CHILE

\begin{tabular}{|l|c|}
\hline CARRERA & NÚMERO DE TESIS \\
\hline Administración & 1 \\
\hline Administración de Empresas de Turismo & 3 \\
\hline Agronomía & 9 \\
\hline Antropología & 5 \\
\hline Arquitectura & 1 \\
\hline
\end{tabular}




\begin{tabular}{|l|c|}
\hline CARRERA & NÚMERO DE TESIS \\
\hline Biología marina & 3 \\
\hline Enfermería & 3 \\
\hline Ingeniería en Acuicultura & 5 \\
\hline Ingeniería Civil en Obras Civiles & 2 \\
\hline Ingeniería en Construcción & 4 \\
\hline Ingeniería en Conservación de Rec. Naturales & 1 \\
\hline Ingeniería Comercial & 7 \\
\hline Ingeniería Forestal & 1 \\
\hline Ingeniería Naval & 3 \\
\hline Licenciatura en Ciencias & 1 \\
\hline Licenciatura en Educación & 10 \\
\hline Medicina Veterinaria & 1 \\
\hline Obstetricia y Puericultura & 2 \\
\hline Pedagogía en Educación Básica & 2 \\
\hline Pedagogía en Historia y Geografía & 8 \\
\hline Periodismo & 1 \\
\hline Química y Farmacia & 5 \\
\hline Turismo & \\
\hline
\end{tabular}

Fuente: Elaboración propia

Tabla 5

NÚMERO DE TESIS SEGÚN PROGRAMA DE POST GRADO DE LA UNIVERSIDAD AUSTRAL DE CHILE

\begin{tabular}{|l|c|}
\hline PROGRAMA DE POST GRADO & NÚMERO DE TESIS \\
\hline Magister en Ciencias del Suelo & 1 \\
\hline Magister en Desarrollo Rural & 4 \\
\hline Magister en Economía y Gestión Regional & 6 \\
\hline
\end{tabular}

Fuente: Elaboración propia

Tabla 6

DISTRIBUCIÓN TEMPORAL DE LAS TESIS.

\begin{tabular}{|l|c|c|}
\hline GRUPO TEMÁTICO & PERIODO (AÑOS) & NÚMERO DE TESIS \\
\hline Ciencias de la Tierra & $1970-2000$ & 2 \\
\hline & $2001-2005$ & 3 \\
\hline
\end{tabular}




\begin{tabular}{|l|c|c|}
\hline GRUPO TEMÁTICO & PERIODO (AÑOS) & NÚMERO DE TESIS \\
\hline Ciencias de la Tierra & $2006-2010$ & 3 \\
\cline { 2 - 3 } & $2011-2015$ & 4 \\
\hline Ciencias de la Vida & $1970-2000$ & 5 \\
\cline { 2 - 3 } & $2001-2005$ & 8 \\
\cline { 2 - 3 } & $2006-2010$ & 18 \\
\cline { 2 - 3 } & $2011-2015$ & 10 \\
\hline Territorio y Cs. de la Tierra & $1970-2000$ & 7 \\
\cline { 2 - 3 } & $2001-2005$ & 18 \\
\cline { 2 - 3 } & $2006-2010$ & 10 \\
\cline { 2 - 3 } & $2011-2015$ & 19 \\
\hline
\end{tabular}

Fuente: Elaboración propia

Es necesario tener en consideración que estos resultados reflejan la combinación de un conjunto de factores decisionales de parte tanto del estudiante tesista como del profesor guía o tutor. Por lo tanto, dependerán de la línea de trabajo del tutor y del apoyo que el proyecto pueda encontrar para su financiamiento.

Un efecto de este tipo de influencias puede explicar el incremento de las investigaciones a partir del año 2005, coincidiendo con el inicio de actividades del Centro de Investigación en Ecosistemas de la Patagonia (CIEP), que ha servido de plataforma para la ejecución de proyectos por parte de investigadores y estudiantes de la Universidad. Lo mismo puede señalarse respecto al rol permanente de desarrollo de investigaciones en el Centro de la Trapananda, en el Campus Patagonia de la Universidad Austral. De igual manera puede suponerse un efecto dinamizador por parte de los programas FIC Regional (Fondo de Innovación para la Competitividad), junto a otros instrumentos de apoyo a la investigación de base regional.

\subsection{Distribución espacial de las tesis y memorias de título o grado}

Con el propósito de evaluar la cobertura espacial que presentan los trabajos de titulación analizados en este artículo, se presenta en la figura 2 su distribución en el territorio regional, según la información de coordenadas extraída de los propios trabajos. Debido a la concentración de trabajos en una misma localización, se ha optado por señalar el número de tesis realizadas en cada caso.

Una situación particular se produce con las tesis cuya área de estudio corresponde a la Región completa. Para este caso se ha determinado un centroide y se han localizado en ese punto las 18 tesis que tienen esta condición. Esto es importante de tener presente para evitar que esta opción cartográfica mueva a error en la interpretación. 
Figura 2

DISTRIBUCIÓN ESPACIAL DE LAS TESIS REALIZADAS EN LA UNIVERSIDAD AUSTRAL DE CHILE

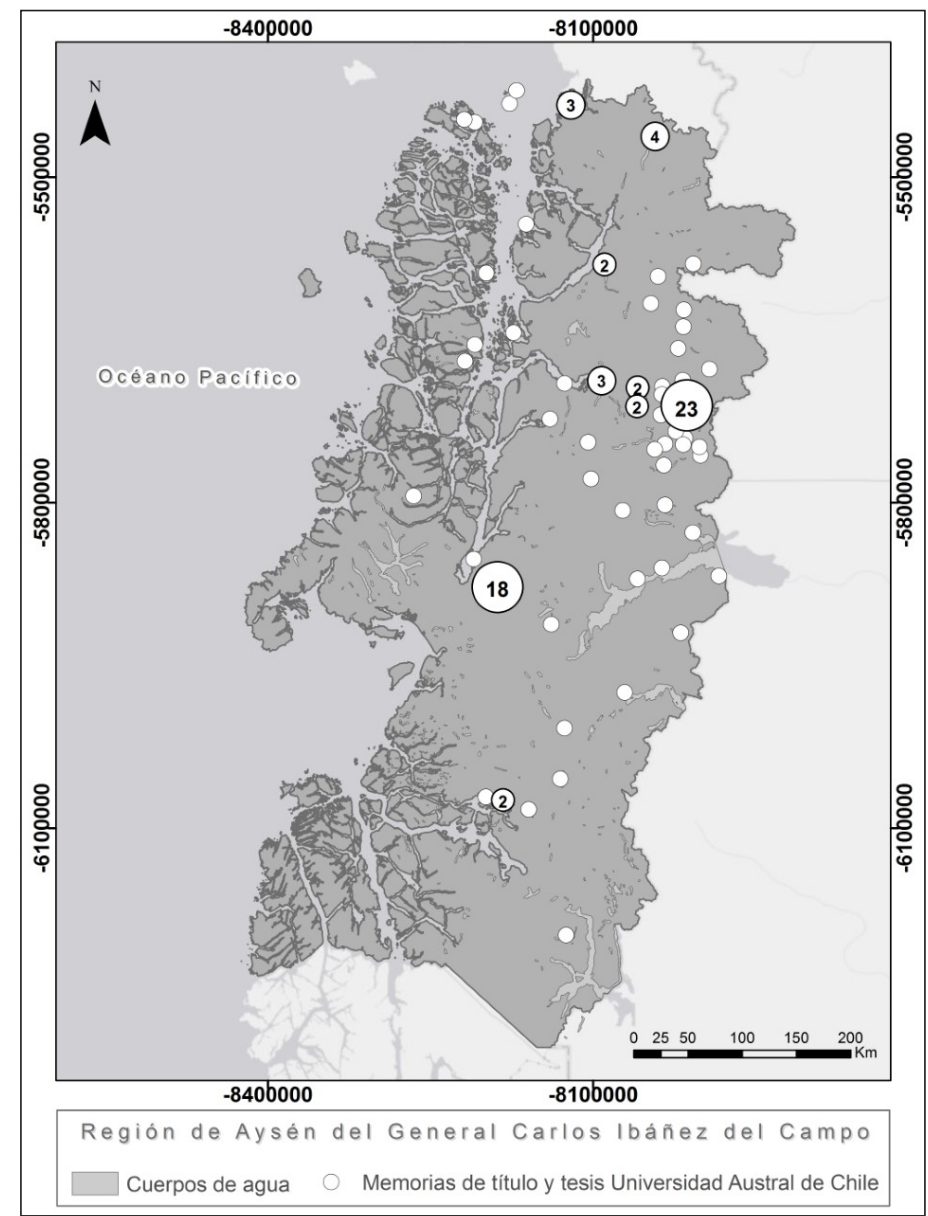

Fuente: Elaboración propia

Dos aspectos principales se deducen de la Figura 2. El primero dice relación con la concentración de trabajos en el eje central de poblamiento, es decir el valle del río Simpson en el cual se emplazan las ciudades de Coyhaique y Puerto Aysén que en conjunto reúnen cerca del $70 \%$ de la población regional. El segundo hecho se refiere al predominio de tesis que se localizan en el sector oriente de la Región, lo que tiene su explicación en que es el área de mayor concentración de actividades económicas a la vez que el sector de mayor habitabilidad.

Para un mayor detalle de la información, la Figura 3 presenta la distribución de las 107 tesis catastradas, divididas en los tres grupos temáticos definidos en la metodología. De 
la información así representada se puede observar, por ejemplo, que de las 18 tesis que cubren la Región en su totalidad, 16 pertenecen al grupo de Territorio y Ciencias Sociales, lo que implica que este tipo de estudios es el que mejor se adecúa al nivel regional de análisis. Otro aspecto que resalta de esta cartografía, es la dispersión de las tesis del grupo Ciencias de la Vida, que cubren espacios litorales e interiores de la Región. En cambio, los trabajos de Ciencias de la Tierra y de Territorio y Ciencias Sociales muestran una tendencia a la concentración en el área centro de la Región, donde se localizan las actividades silvo-agropecuarias por un lado y también se concentra la población.

\section{Figura 3 \\ DISTRIBUCIÓN ESPACIAL DE LAS TESIS, SEGÚN GRUPO TEMÁTICO A QUE FUERON ADSCRITAS}

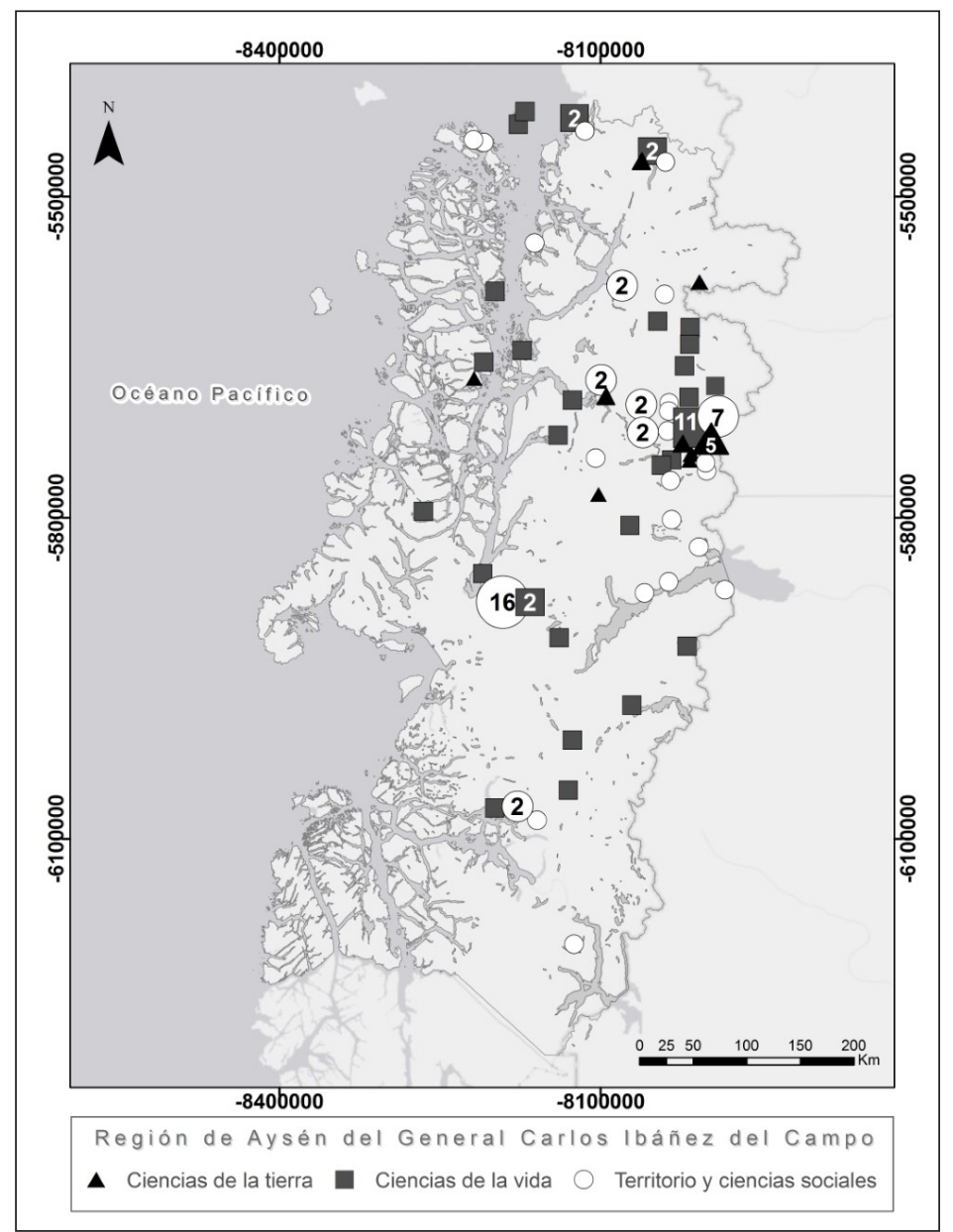

Fuente: Elaboración propia 
En un mayor detalle de la distribución de las tesis, se hace un análisis por sectores de la Región. En primer lugar, se advierte una concentración en el sector norte de la Región de Aysén. Específicamente se trata de una concentración en el sector litoral, de tesis del grupo Ciencias de la Vida, principalmente de Biología Marina, situación que se repite en las que se desarrollan en algunos sectores interiores con vocación para pesca deportiva.

Respecto al sector central de la región, se aprecia una alta concentración en el eje Coyhaique - Puerto Aysén, donde se localizan 33 tesis, es decir casi un tercio del total de investigaciones realizadas en la Región. Entre ellas hay una alta proporción de tesis en el grupo de Territorio y Ciencias Sociales, lo que se condice con la referida concentración de población y actividades urbanas. En este sector se emplazan las tesis de tipo regional, las que como ya se mencionó son predominantemente del grupo de Territorio y Ciencias Sociales.

Finalmente, el sector sur de la Región es el que reúne la menor cantidad de tesis, lo que puede tener explicación en la menor accesibilidad que presentan los distintos sectores de este territorio. Un aspecto que puede confirmar esto es el hecho de que las tesis muestran una distribución que sigue la Ruta 7 (Camino Longitudinal Austral), entre la localidad de Cochrane y Caleta Tortel. Las tesis son preferentemente del grupo de Ciencias de la Vida, aunque en Caleta Tortel y Villa O'Higgins se registran trabajos de Territorio y Ciencias Sociales.

\subsection{Potencial turístico de las tesis y memorias de título o grado}

Para cumplir con el objetivo central de este estudio, se ha evaluado el potencial que cada tesis o memoria de título tiene, para sustentar proyectos de turismo y específicamente de turismo científico. Como ya se señaló los criterios para esta evaluación fueron principalmente, la temática del estudio, su localización y el tipo de información que aporta.

Como resultado de esta evaluación se identificó un total de 25 tesis que presentan este potencial a juicio de los autores de este artículo. Ellas se presentan en la Tabla 7 , la que permite observar el área científica a la que pertenecen. Como se puede apreciar, Biología Marina y Turismo concentran la mayoría de estas tesis (14 de 25). En el caso de Biología Marina, se trata de tesis que aportan conocimientos que permiten continuar líneas de investigación de interés, tanto para la Región, como para el desarrollo científico. Las tesis de Turismo por su parte, aportan antecedentes del grado de desarrollo de esta actividad, así como de la naturaleza de las potencialidades que tiene el territorio para soportar este tipo de estudios. Respecto a los grupos temáticos, se reparten casi en partes iguales entre Ciencias de la Vida (12 tesis) y Territorio y Ciencias Sociales (13 tesis).

Con el fin de analizar la distribución espacial de estas tesis, se ha preparado el mapa de la Figura 4 en el cual se puede observar una tendencia interior en la distribución, además de una concentración relativa en el sector centro y sur de la Región. En el sector norte específicamente, se encuentran dos tesis relacionadas con avistamiento de ballenas, temática de gran relevancia para planes de desarrollo de actividades turísticas 
Tabla 7

TESIS CON POTENCIALIDAD PARA EL TURISMO, SEGÚN ÁREA CIENTÍFICA A QUE ADSCRIBIERON

\begin{tabular}{|l|c|}
\hline Área Científica & $\begin{array}{c}\text { Número de } \\
\text { Tesis }\end{array}$ \\
\hline Biología Marina & 8 \\
\hline Turismo & 6 \\
\hline Economía & 3 \\
\hline Antropología & 2 \\
\hline Ecología & 2 \\
\hline Arquitectura & 1 \\
\hline Botánica & 1 \\
\hline Planificación Territorial & 1 \\
\hline Zoología & 1 \\
\hline
\end{tabular}

Fuente: Elaboración propia

en el golfo de Corcovado y áreas adyacentes y que tienen una fuerte implicancia para proyectos de turismo científico.

Otro conjunto de tesis consideradas de importancia para el turismo son los siete trabajos en el ámbito de la pesca deportiva, principalmente desde la biología marina, pero también con aportes desde la economía y el turismo. Estas tesis se distribuyen a lo largo y ancho del territorio regional, desde la cuenca del río Cisnes por el norte, hasta la cuenca del río Baker por el sur.

Entre las seis tesis relativas a temas turísticos y que contienen elementos que pueden aportar al desarrollo de turismo científico, destacan las dedicadas a patrimonio como la que se refiere al Museo de Sitio de Puerto Cristal, y las que tienen implicancias potenciales en desarrollo de proyectos locales como la de Caleta Tortel y de Villa O'Higgins.

Desde la ecología se identifican cuatro tesis que pueden aportar al turismo científico, dos de ellas se refieren a estudios en huemules y otras dos a fauna en la Reserva Nacional Trapananda, al nor-este de Coyhaique y en la reserva Nacional Lago Jeinimeni, en la cuenca del lago General Carrera. Se puede incluir además en el ámbito del estudio en Reservas Nacionales una tesis de economía que aborda el desarrollo local en Cerro Castillo, localidad directamente vinculada a la Reserva Nacional del mismo nombre. 


\section{Figura 4}

DISTRIBUCIÓN ESPACIAL DE LAS TESIS CON POTENCIALIDAD

\section{PARA EL TURISMO}

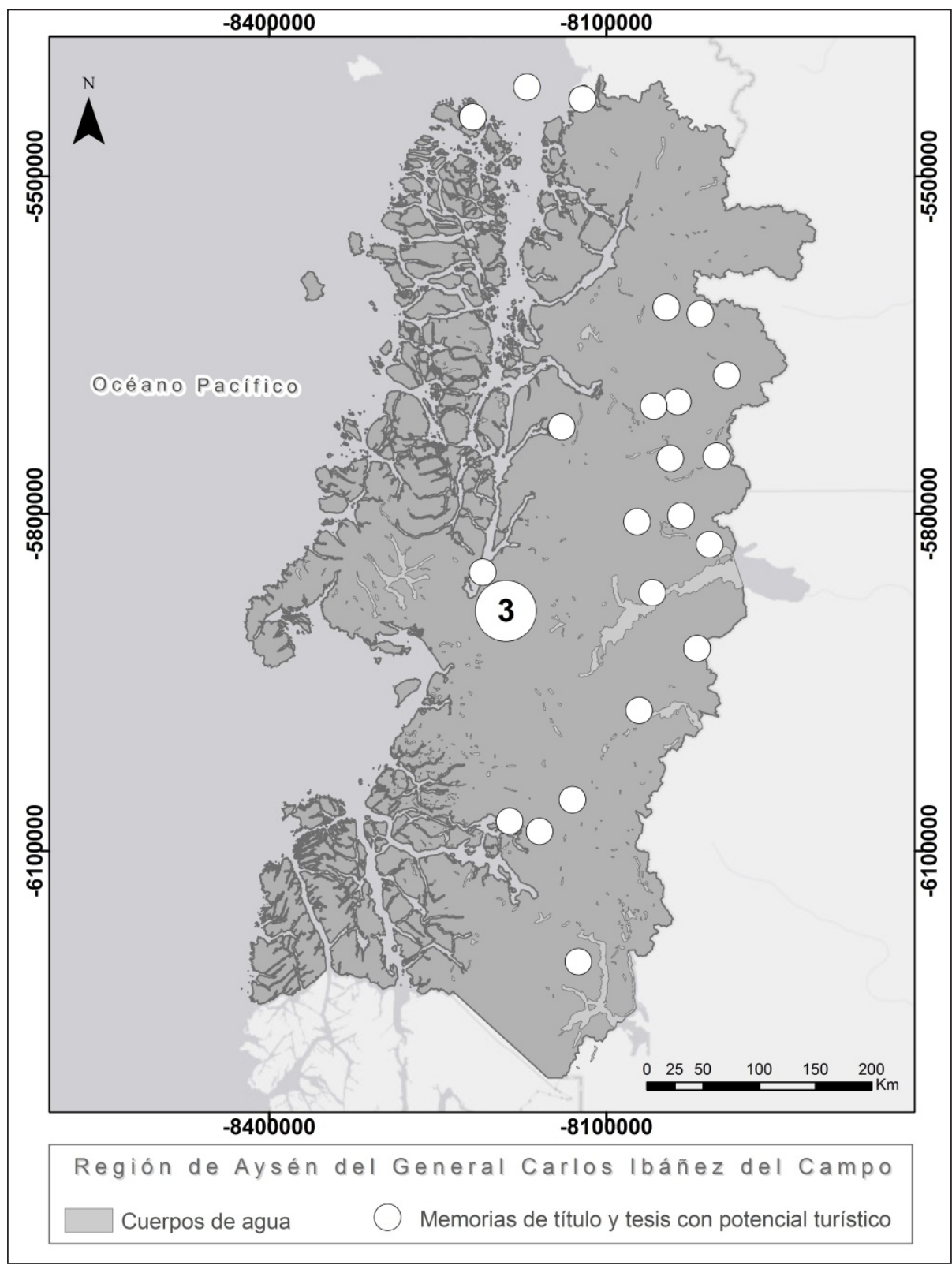

Fuente: Elaboración propia 


\section{CONCLUSIONES}

La Universidad Austral de Chile a través del trabajo de los estudiantes tesistas de pre y post grado, ha realizado un aporte al conocimiento científico de la Región de Aysén desde diversas disciplinas. Destacan los aportes realizados por las tesis en Biología marina, tanto respecto a recursos pesqueros marinos, como en lo relativo a la pesca deportiva, tema de importancia en esta Región. Esta ha sido la carrera que ha aportado con el mayor número de tesis a esta recopilación.

Del análisis de las 107 tesis catastradas en el sistema de bibliotecas de la Universidad, 25 han podido ser calificadas como con potencialidad para apoyar y soportar proyectos de turismo científico, ya sea porque entregan los antecedentes necesarios como para iniciar o continuar investigaciones, o bien por tratarse de estudios desarrollados en alguna de las áreas silvestres protegidas de la Región. Entre ellas se encuentran 7 de Biología Marina, 6 de Turismo y 4 del área de la Ecología.

En este análisis de las tesis de la Universidad Austral de Chile, se aprecia una tendencia a la concentración en el sector central y oriental de la Región. Quedan espacios relativamente "vacíos" desde este punto de vista, en la costa, específicamente en el archipiélago al oeste del canal Moraleda, y en el sector sur de la Región.

Otro déficit detectado dice relación con tesis en Ciencias de la Tierra, por cuanto las 9 tesis que se encontraron tienen relación principalmente con temas agronómicos. Se carece de estudios en ciencias básicas como geología, meteorología, hidrología y geomorfología, por ejemplo. Tampoco se encontró tesis en el ámbito de la arqueología, que también es uno de los temas importantes para el turismo científico a nivel mundial.

La concentración observada en el sector central, especialmente en el eje Coyhaique Puerto Aysén y alrededores, deja por una parte en claro que esta área dispone de suficientes estudios que pueden apoyar proyectos de turismo científico, aunque con la limitante de que posiblemente podrían encontrarse algunos temas relativamente agotados. Por otra parte, la ausencia de estudios en el área litoral y sur, dejan un amplio campo de estudio para la realización de tesis en las diferentes carreras de la Universidad.

En general se puede concluir que el esfuerzo de estudiantes y profesores para desarrollar estos estudios, constituye un aporte significativo a la existencia de información y conocimiento sobre este territorio. Queda por analizar la existencia de otras fuentes de información científica, lo que hará posible disponer de una mayor base de antecedentes para la planificación del turismo científico en la Región de Aysén.

\section{BIBLIOGRAFÍA}

BOURLON, F. y MAO, P. (2011). "Las formas del turismo científico en Aysén, Chile". Gestión Turística, ${ }^{\circ}$ 15, pp. 74-98.

BOURLON, F., SOTO, D. y PASTRIAN, I. (Eds.). (2016). La ruta de los archipiélagos patagónicos: Guía para el turismo científico Región de Aysén. CIEP-Chile.

BARÓMETRO OMT DEL TURISMO MUNDIAL. (2017). El turismo internacional mantiene un crecimiento sostenido pese a las dificultades. Enero 2017 Disponible en http://www2.unwto.org/es 
CHILE. SUBSECRETARÍA DE TURISMO. (2015): Plan Nacional de Desarrollo Turístico Sustentable. Disponible en http://www.subturismo.gob.cl/.

COMANESCU, L. y DOBRE, R. (2009): "Inventoring, evaluating and tourism valuating. The geomorphosites from the central sector of the Ceahlau National Park". GeoJournal of Tourism and Geosites, vol. 1, n 3, pp. 86-96.

CYNARSKI, W. y KUBALA, K. (2017): "Congress in Rio Maior: the particular example of martial arts tourism and scientific tourism". IDO Movement for CultureJournal of Martial Arts Anthropology. vol. 17, $\mathrm{n}^{\circ}$ 2, pp. 34-40.

GARCÍA, M. y MARTÍNEZ, O. (2017): “Turismo científico y ciudades del futuro". International Journal of Scientific Management and Tourism., vol. 3, $\mathrm{n}^{\mathrm{o}}$ 1, pp. 123130

GOBIERNO DE CHILE. (s.f. a). Chile. Estrategia Nacional de Turismo 2012-2020. Disponible en http://www.subturismo.gob.cl/

GOBIERNO DE CHILE (s.f. b). Política Regional de Turismo de Aysén. Subsecretaría de Desarrollo Regional - Aysén Gobierno Regional. 28 páginas. Disponible en http:// www.goreaysen.cl/

GIORDANO, E., GIARDINO, M., PEROTTI, L, GHIRALDI, L. y PALOMBA, M. (2016): "Following the Tracks of Charlemagne in the Cottian Alps. The Cultural and Geological Heritage of the Franks Trail (Susa Valley, Piemonte, NW Italy)". Geoheritage, vol. 8, n 4, pp. 293-300.

KUBALIKOVÁ, L. (2013): "Geomorphosite assessment for geotourism purposes". Czech Journal of Tourism, vol. 2, $\mathrm{n}^{\circ}$ 2, pp. 80-104.

MIGOŃ, P. y PIJET-MIGOŃ, E. (2016): “Overlooked Geomorphological Component of Volcanic Geoheritage-Diversity and Perspectives for Tourism Industry, Pogórze Kaczawskie Region, SW Poland". Geoheritage, vol. 8, n 4, pp. 333-350.

PARDO, C.J. y NIETO, A. (2015): “Turismo antártico: Naturaleza y ciencia en los confines del turismo contemporáneo". Anales de Geografía de la Universidad Complutense, vol. 35, $\mathrm{n}^{\circ} 1$, pp. 139-168.

PARDO, C.J. y NIETO, A. (2016): “Turismo en la Antártida: precisiones conceptuales, principios generales y perspectivas teóricas". Estudios Geográficos, vol. LXXVII, $\mathrm{n}^{\circ} 280$, pp. 241-273.

PRALONG, J.P. (2005): “A method for assessing tourist potential and use of geomorphological sites”. Geomorphologie: relief, processus, environnement, vol. 11, n 3 , pp. 189-196.

REYNARD, E. (2008): "Scientific research and tourist promotion of geomorphological heritage". Geogr. Fis. Dinam. Quat, n 31, pp. 225-230.

SERNATUR (s.f. a): Informe temporada de turismo 2015 -2016. Periplo. El Barómetro Turístico de la Región de Aysén. Boletín n 1.

SERNATUR (s.f. b): Plan de Acción Región de Aysén del General Carlos Ibáñez del Campo. Sector Turismo. 2014-2018. Servicio Nacional de Turismo, Chile. 58 pp.

SIEBER, L., CYNARSKI, W. y MYTSKAN, T. (2015): "Study trip to Munich. Casus of scientific and martial arts tourism". IDO Movement for Culture. Journal of Martial Arts Anthropology, vol. 4, $\mathrm{n}^{\circ}$ 15, pp. 49-57. 
SILVA, S. (2010): Desarrollo local y alternativas de desarrollo productivo: el impulso de un clúster eco-turístico de la región de Aysén. CEPAL (Comisión Económica para América Latina y el Caribe). Disponible en http://unpan1.un.org/intradoc/groups/ public/documents

TERRITORIO AYSÉN. (2014): Informe Final Consultoría: Desarrollo de Mapas, Catastro y Sistematización de Sitios de Investigación en el Litoral de la Región de Aysén. Centro de investigaciones en Ecosistemas de la Patagonia (CIEP), Banco Interamericano de Desarrollo (BID), Fondo Multilateral de Inversiones (FOMIN). Coyhaique, Chile. 
